\title{
Activity Restriction Among Women With a Short Cervix
}

\author{
William A. Grobman, MD, MBA, Sharon A. Gilbert, MBA, PhD, Jay D. lams, MD, Catherine Y. \\ Spong, MD, George Saade, MD, Brian M. Mercer, MD, Alan T. N. Tita, MD, PhD, Dwight J. \\ Rouse, MD, Yoram Sorokin, MD, Kenneth J. Leveno, MD, Jorge E. Tolosa, MD, MSCE, John \\ M. Thorp, MD, Steve N. Caritis, MD, J. Peter Van Dorsten, MD, and for the Eunice Kennedy \\ Shriver National Institute of Child Health and Human Development (NICHD) Maternal-Fetal \\ Medicine Units (MFMU) Network ${ }^{\star}$ \\ Departments of Obstetrics and Gynecology, Northwestern University, Chicago, Illinois, The Ohio \\ State University, Columbus, Ohio, University of Texas Medical Branch, Galveston, Texas, Case \\ Western Reserve University-MetroHealth Medical Center, Cleveland, Ohio, University of \\ Alabama at Birmingham, Birmingham, Alabama, Brown University, Providence, Rhode Island, \\ Wayne State University, Detroit, Michigan, University of Texas Southwestern Medical Center, \\ Dallas, Texas, Oregon Health \& Science University, Portland, Oregon, University of North \\ Carolina at Chapel Hill, Chapel Hill, North Carolina, University of Pittsburgh, Pittsburgh, \\ Pennsylvania, and Medical University of South Carolina, Charleston, South Carolina; the George \\ Washington University Biostatistics Center, Washington, DC; and the Eunice Kennedy Shriver \\ National Institute of Child Health and Human Development, Bethesda, Maryland
}

\section{Abstract}

OBJECTIVE-To estimate determinants of and outcomes associated with activity restriction among women with a short cervix.

\begin{abstract}
METHODS-This was a secondary analysis of a randomized trial of 17-a hydroxyprogesterone caproate for prevention of preterm birth among nulliparous women with singleton gestations and cervices less than $30 \mathrm{~mm}$ by midtrimester ultrasonography. Women were asked weekly whether they had been placed on pelvic, work, or nonwork rest. "Any activity restriction" was defined as being placed on any type of rest. Factors associated with any activity restriction were determined and the association between preterm birth and activity restriction was estimated with multivariable logistic regression.
\end{abstract}

RESULTS-Of the 657 women in the trial, 646 (98\%) responded to questions regarding activity restriction. Two hundred fifty-two (39.0\%) were placed on any activity restriction at a median of 23.9 weeks (interquartile range 22.6-27.9 weeks).Women on activity restriction were older, more

\footnotetext{
(C) 2013 by The American College of Obstetricians and Gynecologists. Published by Lippincott Williams \& Wilkins. Corresponding author: William A. Grobman, MD, MBA, 250 East Superior Street, Suite 05-2175, Chicago, IL 60611; wgrobman@northwestern.edu.

For a list of other members of the NICHD MFMU, see the Appendix online at http://links.lww.com/AOG/A376.

Dr. Spong, and Dr. Rouse, Associate Editors of Obstetrics \& Gynecology, were not involved in the review or decision to publish this article.

Presented at the Society for Maternal-Fetal Medicine meeting, February 11-16, 2013, San Francisco, California.

Financial Disclosure

The authors did not report any potential conflicts of interest.
} 
likely to have private insurance, less likely to be Hispanic, had a shorter cervical length, and were more likely to have funneling and intra-amniotic debris. Preterm birth at less than 37 weeks of gestation was more common among women placed on activity restriction (37\% compared with $17 \%, P<.001)$. After controlling for potential confounding factors, preterm birth remained more common among those placed on activity restriction (adjusted odds ratio $2.37,95 \%$ confidence interval 1.60-3.53). Results were similar for preterm birth at less than 34 weeks of gestation.

CONCLUSION-Activity restriction did not reduce the rate of preterm birth in asymptomatic nulliparous women with a short cervix.

Preterm birth remains a leading cause of perinatal morbidity and mortality. ${ }^{1}$ In an effort to reduce its frequency, investigators have studied a wide variety of prophylactic and therapeutic strategies such as antibiotics, intravenous fluids, and both short-term and longterm tocolysis. None of these strategies, however, has been proven to reduce the frequency of preterm birth. ${ }^{2}$

Similarly, activity restriction has been widely studied for preterm birth prophylaxis and treatment. ${ }^{3-5}$ Activity restriction takes many forms, including pelvic rest, work stoppage, and bed rest. None of these variations is known to be efficacious, and each may be associated with adverse social, economic, and health consequences. ${ }^{6,7}$

Two randomized trials have demonstrated that vaginal progesterone, when administered to women found to have a short cervix on transvaginal ultrasonography, reduces the frequency of preterm birth. ${ }^{8,9}$ Accordingly, some have advocated for midtrimester cervical length screening for all pregnant women. ${ }^{10}$ Others, however, have urged caution, noting the possibility of unanticipated, potentially unhelpful, and costly strategies that may be enacted in response to the finding of a short cervix. ${ }^{11}$ This study was designed to estimate the extent to which women with a short cervix have activity restriction recommended and to estimate the association between this intervention and preterm birth.

\section{MATERIALS AND METHODS}

The Short Cervix and Nulliparity trial was a randomized placebo-controlled trial in which asymptomatic nulliparous women with singleton gestations and a cervices less than $30 \mathrm{~mm}$ (ie, less than the tenth percentile), as measured by midtrimester transvaginal ultrasonography, received either weekly intramuscular 17-a hydroxyprogesterone caproate or placebo. The simple urn method of randomization, with stratification according to clinical center, was used by the data coordinating center to create the computer-generated randomization sequence. ${ }^{12}$ Women with a known last menstrual period were dated by their ultrasonographic examination if a discrepancy existed of more than 7 days at less than 20 weeks of gestation and more than 14 days between 20 and 22 6/7 weeks of gestation. If the last menstrual period was unknown, gestational age was established by the first ultrasonographic examination using the standard method of ultrasonographic gestational age determination at that institution. Full details of the study protocol have been previously reported. ${ }^{12}$ 
The present study is a secondary analysis of data from the Short Cervix and Nulliparity trial. During the weekly study visits for the trial, participants were queried as to whether their health care provider had recommended any of these forms of activity restriction: pelvic rest, reduction of work activity, or reduction of nonwork activity. Those who did not answer these questions were excluded from further analysis. "Pelvic rest" was defined as prohibition of sexual activity. Those participants who had recommended reductions in work or nonwork activity were further categorized according to whether the reduction in this activity was partial (the recommendation allowed some work or nonwork activity to continue) or complete (the recommendation was for all work and nonwork activity to cease). The frequencies of different variations of activity restriction, and the median gestational age at which they were recommended, were determined. Those women who had reductions in either pelvic, work, or nonwork activity were classified as having had "any" activity restriction.

Patient factors (both demographic and ultrasonographic) associated with any activity restriction were estimated in univariable analysis. All ultrasonographic findings were derived from the initial screening ultra-sonogram. We then explored the association between activity restriction and preterm birth at less than 37 and less than 34 weeks of gestation. The association was first estimated in univariable analysis and then in a multivariable logistic regression. In addition to the activity restriction variable, other independent variables placed in the multivariable regression were treatment group (ie, whether a patient received 17-a hydroxyprogesterone caproate or placebo) and any patient factors that had been identified in univariable analysis as being associated at $P<.05$ with having activity restriction recommended. The interactions of activity restriction with 1) treatment group; 2) cervical length; and 3) gestational age at screening also were assessed. In addition, the univariable and multivariable analyses were repeated with "activity restriction" being defined only according to whether women were recommended to forego work or nonwork activities (and without regard to pelvic rest).

The Wilcoxon rank-sum test was used to compare continuous variables, the $\chi^{2}$ was used to compare categorical variables, and the Breslow-Day test was used to test for interaction and homogeneity of the odds ratio in stratified analyses. Multivariable analyses were performed with multiple logistic regression with odds ratios (ORs) and 95\% confidence intervals (CIs) reported. All tests were two-tailed and $P<.05$ was used to define statistical significance. No adjustments were made for multiple comparisons. All analyses were performed using SAS 9.2 (SAS Institute, Cary, NC) with Figure 1 constructed with the use of R (http://www.rproject.org). The study was approved by the internal review boards at all participating centers.

\section{RESULTS}

Of the 657 women who participated in the randomized trial, $646(98 \%)$ responded to questions regarding activity restriction. The frequencies of different forms of activity restriction, and the median gestational ages at which they were first recommended, are presented in Table 1. Nearly forty percent of women with a short cervix were prescribed some form of activity restriction, most commonly in the midsecond to early third trimester, 
soon after the diagnosis of a short cervix was made. As illustrated by Figure 1, various combinations of this restriction were used, although most commonly (171 of 252 [68\%]), all three types were combined.

Women who had any activity restriction recommended, compared with those who did not, differed in many of their demographic characteristics and trans-vaginal ultrasonographic findings (Table 2). Specifically, women who received recommendations for activity restriction were older $(P<.001)$, were more likely to have private insurance $(P=.01)$, and were less likely to be Hispanic white $(P<.001)$. These women also had shorter cervical lengths and were more likely to have cervical funneling or intra-amniotic debris, as assessed by transvaginal ultrasonography.

Preterm birth at less than 37 weeks of gestation was significantly more common among women placed on any activity restriction (37\% compared with $17 \%, P<.001$; OR $2.91,95 \%$ CI 2.0-4.21). No significant interactions between treatment with 17-a hydroxyprogesterone caproate and activity restriction, cervical length less than $15 \mathrm{~mm}$ and activity restriction, or gestational age at screening less than 20 weeks of gestation and activity restriction were found and therefore these interactions were not assessed further (Breslow-Day for treatment group: $P=.67$ at 37 weeks of gestation, $P=.93$ at 34 weeks of gestation; for cervical length: $P=.70$ at 37 weeks of gestation, $P=.82$ at 34 weeks of gestation; for gestational age at screening: $P=.11$ at 37 weeks of gestation, $P=.40$ at 34 weeks of gestation). After controlling for treatment group and for the noted demographic and ultrasonographic differences among those with and without activity restriction, preterm birth less at than 37 weeks of gestation remained significantly more common among those placed on any activity restriction (adjusted OR 2.37, 95\% CI 1.60-3.53; Table 3). Similarly, preterm birth at less than 34 weeks of gestation was significantly more common among women placed on any activity restriction (adjusted OR 2.28, 95\% CI 1.36-3.80). Results were similar when only women prescribed limitation of work and nonwork activities were included in the activity restriction group (less than 37 weeks of gestation: adjusted OR 2.44, 95\% CI 1.63-3.65; less than 34 weeks of gestation: adjusted OR 2.70, 95\% CI 1.62-4.52).

\section{DISCUSSION}

This analysis reveals that some form of activity restriction was prescribed for more than one in every three nulliparous women who were diagnosed with a short cervix. Activity restriction, when it was recommended, was not narrowly confined to one aspect of a woman's life but was used such that it pervaded both work and home life. More than two thirds of women who had activity restricted were recommended to refrain from work, nonwork, and sexual activity. It is also of interest to note that activity restriction was not always more likely to be prescribed to women with characteristics most associated with preterm birth. For example, activity restriction was more common among women with private insurance and less common among Hispanic white women than among non-Hispanic white women. This finding suggests that variation in the use of activity restriction is dependent not only on the perception of risk status, but on social factors as well. 
The use of such interventions would be understandable if they had been demonstrated to reduce the frequency of preterm birth. However, there is no good evidence from randomized trials that demonstrate that any of these forms of activity restriction are associated with improved perinatal outcomes for women at increased risk of preterm birth. ${ }^{13}$ Fox et al ${ }^{14}$ specifically evaluated whether hospitalization was efficacious for women with a cervical length $25 \mathrm{~mm}$ or less. In their retrospective analysis, admission to the hospital was not associated with any decrease in the frequency of preterm delivery. Conversely, there was a trend toward an earlier gestational age at delivery among those women hospitalized.

These results are similar to those of our study, in which women who were prescribed activity restriction were significantly more likely to deliver preterm. This association persisted even after controlling for potential confounding factors. It is possible that this association is the result of unmeasured confounding. The most likely cause for unmeasured confounding is that physicians prescribe bed rest to women whom they perceive to be at greatest risk of preterm birth, and some of these factors were not accounted for in our analysis. Yet we were able to prospectively collect and control for many factors, including demographic information, medical history, and ultrasonographic findings that are known to be associated with preterm birth. These are the same factors that would have been known to physicians and would have been responsible for confounding. Conversely, even if there are other unknown factors that predispose women to preterm birth, these would be unknown to physicians as well and could not be responsible for confounding the observed association.

It is not biologically implausible that activity restriction could result in an increased risk of preterm birth. Activity restriction has been associated with increased stress and anxiety, both of which have been associated with an increased risk for adverse pregnancy outcomes, including preterm and low-birth-weight deliveries, in multiple observational studies. ${ }^{15-17}$ Emotional ramifications are not the only consequence of activity restriction, which also has been associated with an increased chance of venous thromboembolism, bone loss, deconditioning, and financial difficulties. ${ }^{6,7}$ Even if there were to be no greater chance of preterm birth with activity restriction, such potential complications underscore that this intervention should be shown to be of benefit before it is used routinely for preterm birth prophylaxis.

This study was performed among nulliparous women with a cervix less than $30 \mathrm{~mm}$. Accordingly, the results cannot be generalized to women who are at higher risk of preterm delivery for other reasons. Also, compliance with activity restriction was not monitored, and we cannot know whether the fidelity with which women followed their physicians' recommendations was associated with the ultimate outcome.

Our data are relevant for the present debate regarding whether transvaginal ultrasonographic cervical length screening during the second trimester should be routinely incorporated into obstetric care. Support for such an approach comes from two cost-effectiveness analyses, which suggested that a universal screening strategy would improve health outcomes and be cost-saving. ${ }^{18,19}$ However, neither analysis incorporated the potential for unexpected consequences from a screening program, including the frequent use of activity restriction for women found to have a short cervical length. Use of such restriction will diminish both the 
financial advantages and health effectiveness of screening, even if the increased risk of preterm birth associated with activity restriction is disregarded. This analysis suggests that, if a cervical length screening program is to be adopted, and the putative health and economic benefits of such a program are to be realized, clear guidelines regarding appropriate responses to a positive finding should be adopted as well. In addition, the analysis emphasizes the importance of conducting an adequately powered randomized trial for women at increased risk of preterm birth such that level I evidence for the effects of activity restriction can be obtained.

\section{Acknowledgments}

Supported by grants from the Eunice Kennedy Shriver National Institute of Child Health and Human Development (NICHD) (HD21410, UL1 RR024153, UL1 TR000005; HD27869, HD27915, HD27917, HD34116, HD34208, 5UL1RR025764, HD36801, HD40500, HD40512, HD40544, M01 RR00080, UL1 RR024989 (NCRR), HD40545, HD40560, HD40485, HD53097, HD53118) and does not necessarily represent the official views of the National Center for Research Resources, NICHD, or National Institutes of Health.

The authors thank Gail Mallett, RN, BSN, CCRC, and Cynthia Milluzzi, RN, for protocol development and coordination between clinical research centers; and Elizabeth Thom, $\mathrm{PhD}$, for protocol and data management and statistical analysis.

\section{References}

1. Spong CY. Prediction and prevention of recurrent spontaneous preterm birth. Obstet Gynecol. 2007; 110:405-415. [PubMed: 17666618]

2. Goldenberg RL. The management of preterm labor. Obstet Gynecol. 2002; 100:1020-1037. [PubMed: 12423870]

3. Hobel CJ, Ross MG, Bemis RL, Bragonier JR, Nessim S, Sandhu M, et al. The West Los Angeles Preterm Birth Prevention Project. I. Program impact on high-risk women. Am J Obstet Gynecol. 1994; 170:54-62. [PubMed: 8296845]

4. Elliott JP, Miller HS, Coleman S, Rhea D, Abril D, Hallbauer K, et al. A randomized multicenter study to determine the efficacy of activity restriction for preterm labor management in patients testing negative for fetal fibronectin. J Perinatol. 2005; 25:626-630. [PubMed: 16107874]

5. Fox NS, Gelber SE, Kalish RB, Chasen ST. The recommendation for bed rest in the setting of arrested preterm labor and premature rupture of membranes. Am J Obstet Gynecol. 2009; 200:165.e1-166.el. [PubMed: 19019329]

6. Sciscione AC. Maternal activity restriction and the prevention of preterm birth. Am J Obstet Gynecol. 2010; 202:232.e1-235.el. [PubMed: 19766979]

7. Maloni JA. Lack of evidence for prescription of antepartum bed rest. Expert Rev Obstet Gynecol. 2011; 6:385-393. [PubMed: 22140399]

8. Fonseca EB, Celik E, Parra M, Singh M, Nicolaides KH. Progesterone and the risk of preterm birth among women with a short cervix. N Engl J Med. 2007; 357:462-469. [PubMed: 17671254]

9. Hassan SS, Romero R, Vidyadhari D, Fusey S, Baxter JK, Khandelwal M, et al. Vaginal progesterone reduces the rate of preterm birth in women with a sonographic short cervix: a multicenter, randomized, double-blind, placebo-controlled trial. Ultrasound Obstet Gynecol. 2011; 38:18-31. [PubMed: 21472815]

10. Campbell S. Universal cervical length screening and vaginal progesterone prevents early preterm birth, reduces neonatal mortality, and is cost saving: doing nothing is no longer an option. Ultrasound Obstet Gynecol. 2011; 38:1-9. [PubMed: 21713990]

11. Parry S, Simhan H, Elovitz M, Iams J. Universal maternal cervical length screening during the second trimester: pros and cons of a strategy to identify women at risk of spontaneous preterm delivery. Am J Obstet Gynecol. 2012; 207:101-106. [PubMed: 22633270] 
12. Grobman WA, Thom EA, Spong CY, Iams JD, Saade GR, Mercer BM, et al. 17 alphahydroxyprogesterone caproate to prevent prematurity in nulliparas with cervical length less than 30 mm. Am J Obstet Gynecol. 2012; 207:390.e1-398.el. [PubMed: 23010094]

13. Sosa C, Althabe F, Belizán JM, Bergel E. Bed rest in singleton pregnancies for preventing preterm birth. The Cochrane Database of Systematic Reviews. 2010; (Issue 1) Art. No.: CD003581.

14. Fox NS, Jean-Pierre C, Predanic M, Chasen ST. Does hospitalization prevent preterm delivery in the patient with a short cervix? Am J Perinatol. 2007; 24:49-53. [PubMed: 17195150]

15. Borders AE, Grobman WA, Amsden LB, Holl JL. Chronic stress and low birth weight neonates in a low-income population of women. Obstet Gynecol. 2007; 109:331-338. [PubMed: 17267833]

16. Coussons-Read ME, Lobel M, Carey JC, Kreither MO, D’Anna K, Argys L, et al. The occurrence of preterm delivery is linked to pregnancy-specific distress and elevated inflammatory markers across gestation. Brain Behav Immun. 2012; 26:650-659. [PubMed: 22426431]

17. Catov JM, Abatemarco DJ, Markovic N, Roberts JM. Anxiety and optimism associated with gestational age at birth and fetal growth. Matern Child Health J. 2010; 14:758-764. [PubMed: 19697113]

18. Cahill AG, Odibo AO, Caughey AB, Stamilio DM, Hassan SS, Macones GA, et al. Universal cervical length screening and treatment with vaginal progesterone to prevent preterm birth: a decision and economic analysis. Am J Obstet Gynecol. 2010; 202:548.e1-548.el. [PubMed: 20079888]

19. Werner EF, Han CS, Pettker CM, Buhimschi CS, Copel JA, Funai EF, et al. Universal cervical length screening to prevent preterm birth: a cost-effectiveness analysis. Ultrasound Obstet Gynecol. 2011; 38:32-37. [PubMed: 21157771] 


\section{Pelvic rest Work restriction}

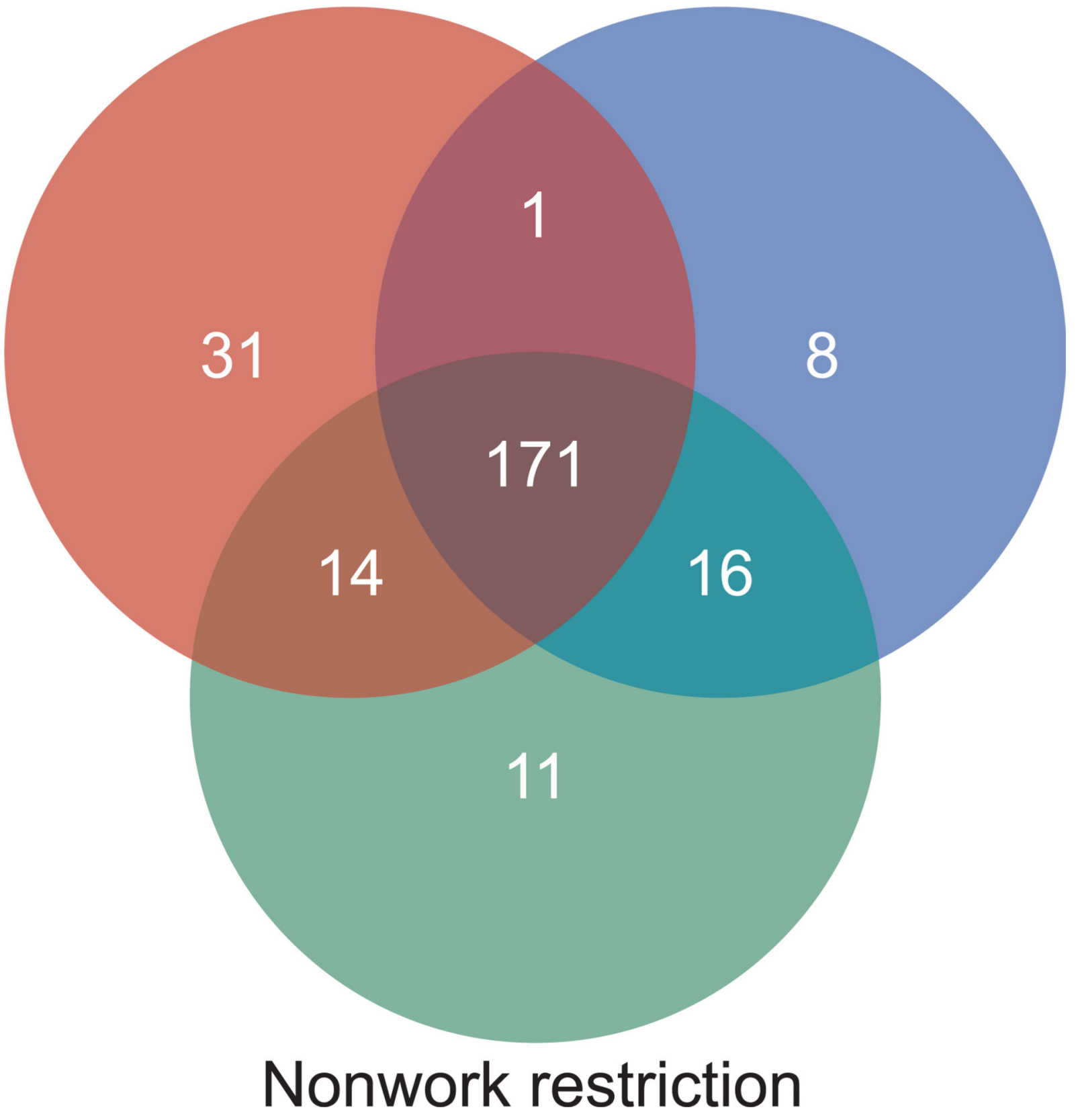

Fig. 1.

Combinations of activity restriction among women with a cervical length less than $30 \mathrm{~mm}$. The numbers show the number of women who experienced each type or combination of restrictions. 


\section{Table 1}

Frequency of and Gestational Age at Initiation of Different Types of Activity Restriction Among Nulliparous Women With a Cervical Length Less Than $30 \mathrm{~mm}$ (N5646)

\begin{tabular}{lcc}
\hline Type of Restriction & Frequency & Estimated Gestational Age (wk) \\
\hline Pelvic rest & $217(33.6)$ & $23.9(22.7,27.7)$ \\
Work restriction & $196(30.3)$ & $24.1(22.8,28.7)$ \\
Partial & $52(8.0)$ & $24.8(22.9,29.8)$ \\
Complete & $144(22.3)$ & $25.0(23.0,29.6)$ \\
Nonwork restriction & $212(32.8)$ & $23.9(22.9,28.2)$ \\
Partial & $89(13.8)$ & $24.4(23.1,28.7)$ \\
Complete & $123(19.0)$ & $24.7(22.9,29.6)$ \\
Work or nonwork restriction & $221(34.2)$ & $23.9(22.9,28.6)$ \\
Work or nonwork restriction or pelvic rest & $252(39.0)$ & $23.9(22.6,27.9)$ \\
\hline
\end{tabular}

Data are $\mathrm{n}(\%)$ or median $\left(25^{\text {th }}, 75^{\text {th }}\right.$ percentile). 


\section{Table 2}

Association Between Demographic and Ultrasonographic Factors and Activity Restriction Among Nulliparous Women With a Cervical Length Less Than $30 \mathrm{~mm}$

\begin{tabular}{lccc}
\hline & $\begin{array}{c}\text { Activity Restriction } \\
(\mathbf{n = 2 5 2})\end{array}$ & $\begin{array}{c}\text { No Activity Restriction } \\
(\mathbf{n = 3 9 4})\end{array}$ & $\boldsymbol{P}$ \\
\hline Treatment group & $131(52.0)$ & $190(48.2)$ & .35 \\
Active & $121(48.0)$ & $204(51.8)$ & \\
Placebo & $21.0(20.0,25.5)$ & $20.0(19.0,23.0)$ & $>.001$ \\
Maternal age (y) & & & .009 \\
Race or ethnicity & $63(25.0)$ & $86(21.8)$ & \\
Non-Hispanic white & $9(3.6)$ & $46(11.7)$ & .03 \\
Hispanic white & $140(55.6)$ & $197(50.0)$ & \\
Black & $3(1.2)$ & $4(1.0)$ & \\
Asian & $37(14.7)$ & $61(15.5)$ & \\
Other & & & \\
Insurance & $66(26.2)$ & $70(17.8)$ & \\
Private & $172(68.3)$ & $292(74.1)$ & \\
Government & $14(5.6)$ & $32(8.1)$ & .007 \\
Self-pay & $20.3(19.4,21.1)$ & $19.7(18.7,20.9)$ & $<.001$ \\
Estimated gestational age at cervical length measurement (wk) & $23.7(19.0,26.5)$ & $26.5(24.0,28.3)$ & $<.001$ \\
Cervical length (mm) & $74(29.4)$ & $77(19.5)$ & .004 \\
Cervical funneling & $14.8(10.7,19.4)$ & $13.0(7.4,19.2)$ & .07 \\
If funneling, length of funnel (mm) & $41(16.3)$ & $35(8.9)$ & \\
Intra-amniotic debris & & & \\
\hline
\end{tabular}

Data are $\mathrm{n}(\%)$ or median $\left(25^{\text {th }}, 75^{\text {th }}\right.$ percentile $)$ unless otherwise specified. 
Table 3

Adjusted Odds Ratios and 95\% Confidence Intervals for the Association Between Patient Factors and Preterm Birth at Less Than 37 Weeks of Gestation

\begin{tabular}{lccc}
\hline Factor & Odds Ratio & 95\% Confidence Interval & $\boldsymbol{P}$ \\
\hline Treatment group & 0.99 & $0.68-1.46$ & .96 \\
Maternal age $(\mathrm{y})^{*}$ & 1.01 & $0.96-1.05$ & .76 \\
Black race & 1.07 & $0.72-1.58$ & .73 \\
Public insurance & 1.60 & $0.92-2.77$ & .10 \\
Estimated gestational age at cervical length measurement $(\mathrm{wk})^{*}$ & 1.02 & $0.89-1.16$ & .78 \\
Cervical length (mm) & 0.93 & $0.90-0.97$ & $<.001$ \\
Cervical funneling & 1.20 & $0.72-2.00$ & .50 \\
Intra-amniotic debris & 0.96 & $0.53-1.73$ & .89 \\
Any activity restriction & 2.37 & $1.60-3.53$ & $<.001$ \\
\hline
\end{tabular}

* Odds ratio per 1 unit change. 